\title{
Increasing applicability of slow-light in molecular aggregate nanofilms with two-exciton dynamics
}

\author{
E. Díaz ${ }^{1 *}$, G. C. Martínez-Calzada ${ }^{1}$, E. Cabrera-Granado ${ }^{2}$, and O. G. Calderón ${ }^{2}$ \\ ${ }^{1}$ GISC, Departamento de Materiales, Universidad Complutense, E-28040 Madrid, Spain \\ ${ }^{2}$ Departamento de Óptica, Universidad Complutense, E-28037 Madrid, Spain \\ *Corresponding author: elenadg@ucm.es
}

Compiled March 25, 2016

\begin{abstract}
We study the slow-light performance in the presence of exciton-exciton interaction in films of linear molecular aggregates at nanometer scale. In particular we consider a four-level model to describe the creation/annihilation of two exciton states. These processes are relevant when high intensity fields are present in the system. Numerical simulations show delays comparable to those obtained for longer propagation distances in other media. Two-exciton dynamics could even drive to larger fractional delays in comparison to the two-level approximation. We also study the influence of disorder usually present in molecular aggregates, whose detrimental effect is remarkably reduced by the two-exciton dynamics. This concludes that slow-light performance is a robust phenomenon in these systems under the increasing complexity of the two exciton dynamics. ( 2016 Optical Society of America
\end{abstract}

OCIS codes: (190.4400) Nonlinear optics, nonlinear optics, materials; (190.5530) Nonlinear optics, pulse propagation and temporal solitons; (230.1150) Optical devices, all-optical devices.

http://dx.doi.org/10.1364/ao.XX.XXXXXX

Slow-light research has shown a great potential in many different applications such as information technology, interferometry or laser physics as a tool to enhance light-matter interactions. However, the overall effect on optical pulses is usually constrained by the necessity of long interacting media. There has been a recent exciting research to provide compact slow-light devices and reduce the needed interacting length without limiting the obtained delay. Group velocities of $c / 100$ have been achieved in $100 \mu \mathrm{m}$ semiconductor waveguides at gigahertz frequencies [1]. More recently, Kim et al. [2] proposed composites doped with metal nanoparticles to obtain fractional delays (ratio between delay and temporal width of the input optical pulse) of 2 for $5-\mu \mathrm{m}$ propagation lengths or even $\sim 40$ for $90 \mu \mathrm{m}$ using a noncollinear pumping scheme. Slow light has also been employed to enhance and control the gain in active semiconductor waveguides [3] which may be used in ultra-compact amplifiers and optical modulators of $\sim 100 \mu \mathrm{m}$ [4] due to the increase in modulation efficiency.

Reducing the slow light devices down to the nanometer scale has been proposed by using coherent population oscillations (CPO) in J-aggregate nanofilms [5]. J-aggregates are molecular assemblies that show coherently coupled transition dipole moments and an absorption band which is narrower and redshifted with respect to the monomer band [6]. They have shown great possibilities in photonic applications thanks to their enhanced nonlinear response and narrow absorption line. This leads, for example, to a coherent coupling of excitons with cavity modes or plasmons in metallic nanostructures [7]. In a previous work, fractional delays up to $\sim 0.5$ were shown with constrained distortion for input pulses of roughly $10 \mathrm{GHz}$-bandwidth in ultrathin films of some tens of nanometers [5]. This work analyzed CPO-based slow light in J-aggregates for optical pulses resonant with the transition to the one-exciton band, while creation and annhilitation of two excitons were not considered. Gain of the probe pulse was also shown, in contrast to the residual absorption observed in other slow-light media such as optical fibers. Moreover, disorder effects typically present in these systems, although producing a detrimental effect on the attainable delay, have a relatively low impact on slow-light performance.

One-to-two exciton transition in J-aggregates were first observed by Fidder et al. [8] in a pump-probe experiment on pseudo-isocyanine-bromide (PIC-Br). More recently, Glaeske et al. [9] showed that such a transition and exciton annihilation play a crucial role in the conditions for achieving optical bistability. Multi-exciton states have also been exploited to coherently control the electromagnetic field inside a cavity [10] and are essential in strong coupling with plasmonic structures [11].

In the present paper, we address how the two-exciton dynamics influence slow light performance of linear J-aggregates nanofilms. We consider an ensemble of linear molecules oriented in the same direction and forming an ultrathin film. Despite being formed by thousands of molecules, only coherent segments of the aggregate with length $N$ are relevant for optical applications due to the states localization in presence of disorder. We model disordered molecular aggregates as an ensemble of homogeneously broadened four-level systems of coherence length $N$. See Ref. [9] for a detailed description of the model. Particularly we simulate three excitonic levels with $|0\rangle,|1\rangle$ and $|2\rangle$ excitons. After the creation of the state $|2\rangle$, the exciton-exciton annihilation occurs by means of a set of electronic-vibrational levels $(3)_{e-v i b}$. 
The excitation then relaxes to the ground vibrational state due to phonon-assisted processes, to finally be transferred again to the $|0\rangle$ or $|1\rangle$ excitonic states. Figure 1 represents the schematics of the model and the pulse propagation through the nanofilm.

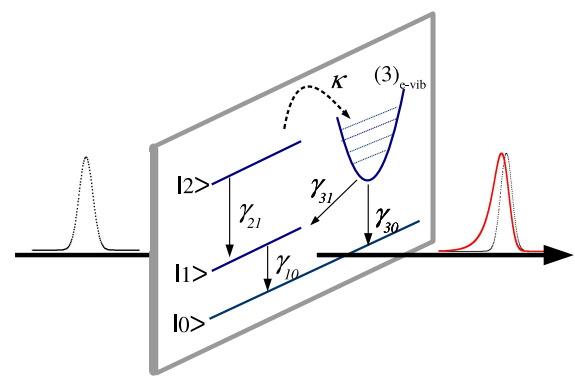

Fig. 1. (Color online) Schematic view of the aggregate nanofilm modeled as an ensemble of four-level systems. Input signal (dashed line) propagating from left to right through the nanofilm results in a delayed output signal (solid line). Pulses are shown according to numerical simulations.

Bloch equations under rotating wave and slowly varying amplitude approximations for this system read:

$$
\begin{aligned}
\dot{\rho}_{00}^{N} & =i d_{10}^{N}\left(\sigma_{10}^{N} E^{*}-\sigma_{10}^{N *} E\right) / 4 \hbar+\gamma_{10}^{N} \rho_{11}^{N}+\gamma_{30}^{N} \rho_{33}^{N} \\
\dot{\rho}_{11}^{N} & =i d_{21}^{N}\left(\sigma_{21}^{N} E^{*}-\sigma_{21}^{N *} E\right) / 4 \hbar-i d_{10}^{N}\left(\sigma_{10}^{N} E^{*}-\sigma_{10}^{N *} E\right) / 4 \hbar \\
& -\gamma_{10}^{N} \rho_{11}^{N}+\gamma_{21}^{N} \rho_{22}^{N}+\gamma_{31}^{N} \rho_{33}^{N} \\
\dot{\rho}_{22}^{N} & =-i d_{21}^{N}\left(\sigma_{21}^{N} E^{*}-\sigma_{21}^{N *} E\right) / 4 \hbar-\left(\gamma_{21}^{N}+w\right) \rho_{22}^{N} \\
\dot{\rho}_{33}^{N} & =-\left(\gamma_{30}^{N}+\gamma_{31}^{N}\right) \rho_{33}^{N}+\kappa \rho_{22}^{N} \\
\dot{\sigma}_{10}^{N} & =\left[i\left(\omega-\omega_{10}^{N}\right)-\Gamma_{10}^{N}\right] \sigma_{10}^{N} \\
& -i d_{10}^{N}\left(\rho_{11}^{N}-\rho_{00}^{N}\right) E / \hbar+i d_{21}^{N} \sigma_{20}^{N} E^{*} / 2 \hbar \\
\dot{\sigma}_{21}^{N} & =\left[i\left(\omega-\omega_{21}^{N}\right)-\Gamma_{21}^{N}-\kappa / 2\right] \sigma_{21}^{N} \\
& -i d_{21}^{N}\left(\rho_{22}^{N}-\rho_{11}^{N}\right) E / \hbar-i d_{10}^{N} \sigma_{20}^{N} E^{*} / 2 \hbar \\
\dot{\sigma}_{20}^{N} & =\left[i\left(\omega-\omega_{10}^{N}\right)+i\left(\omega-\omega_{21}^{N}\right)-\Gamma_{20}^{N}-\kappa / 2\right] \sigma_{20}^{N} \\
& +i d_{21}^{N} \sigma_{10}^{N} E / 2 \hbar-i d_{10}^{N} \sigma_{21}^{N} E / 2 \hbar
\end{aligned}
$$

Here $\omega$ and $E$ are the frequency and slowly varying amplitude of the field, respectively. The population of the energy level $j$ is denoted by $\rho_{j j}^{N}$, while $\sigma_{i j}^{N}$ are the slowly varying amplitudes of the off-diagonal density matrix elements. The latter account for the coherences between the energy levels $(i, j)$. The transition frequency and the dipole moment within those levels of every segment read $\omega_{i j}^{N}$ and $d_{i j}^{N}=d_{i j}^{1} \sqrt{N}$ respectively. Here the superscript 1 refers to single-molecule properties. The relaxation rate due to spontaneous emission is $\gamma_{i j}^{N}=N \gamma_{i j}^{1}$, while $\Gamma_{i j}^{N}$ is the decay of the coherence $\sigma_{i j}^{N}$. Furthermore, $\Gamma_{10}^{N}=\gamma_{10}^{N} / 2+\Gamma$, $\Gamma_{21}^{N}=\gamma_{10}^{N} / 2+\gamma_{21}^{N} / 2+\Gamma$ and $\Gamma_{20}^{N}=\gamma_{21}^{N} / 2+\Gamma$, where $\Gamma$ accounts for pure dephasing processes. The vibronic state $(3)_{e-v i b}$ relaxes to states $|0\rangle$ and $|1\rangle$ with rates $\gamma_{30}^{N}$ and $\gamma_{31}^{N}$ respectively. Last $\kappa$ refers to the exciton-exciton annihilation constant.

Size dispersion of the coherent segments translates into an inhomogeneous broadening affecting the J-band at low temperatures, which mainly gives rise to the fluctuation of the transition energies $\hbar \omega_{10}^{N}$ [12]. Thus, we will substitute all the sizedependent quantities, but $\omega_{10}$, by its mean value in the aggregate and we will remove the index $N$ hereafter. For brevity, we will refer to the field by way of the Rabi frequency defined in units of $\Gamma_{10}$ as $\Omega=d E / \hbar \Gamma_{10}$ from now on, where $d=\sqrt{\left(d_{10}^{2}+d_{21}^{2}\right) / 2}$.

We will first study the response of the system to a sinusoidally modulated signal $\Omega=\Omega_{0}+\Omega_{m} \sin (\delta t)$. Assuming normal and parallel polarization of the incident field to the transition dipole moments of all the aggregates and to the film plane, the equation for the field inside the film takes the form

$$
\Omega=\Omega^{i n}+i \gamma_{R} \sum_{N} p(N)\left(\mu_{10} \sigma_{10}+\mu_{21} \sigma_{21}\right)
$$

Here, the last term is the electric polarization of the disordered molecular aggregates, where $\mu_{10}=d_{10} / d, \mu_{21}=d_{21} / d$ and $p(N)$ refers to the disorder distribution over localization lengths. The parameter $\gamma_{R}=\mu_{0}|d|^{2} N_{0} c \omega L / 2 \hbar \Gamma_{10}$ describes the collective superradiant damping of an ensemble of four-level molecules, being $N_{0}$ the density of localization segments. The transmittance $T$ and the dephasing $\phi$ induced by the film is calculated by the ratio between the output and input signals:

$$
\frac{\Omega_{m}}{\Omega_{m}^{i n}}=T \exp ^{i \phi} .
$$

Thus, the fractional delay is defined as $F=\phi / 2 \pi$.

Let us start analyzing the case of no size dispersion. For simplicity hereafter we consider an incident field resonant with the lower energy transition of the system, $\omega=\omega_{10}$. We take the parameters of PIC-Br as it is one of the most studied Jaggregates. The magnitude of the nearest-neighbor coupling has been established as $J=0.08 \mathrm{eV}$, which gives rise to the transitions energy shift $\omega_{21}-\omega_{10}=3 \pi^{2} \mathrm{~J} / \mathrm{N}^{2} \hbar=0.25 \mathrm{THz}$. We use $\gamma_{10}=1 / 37 \mathrm{ps}^{-1}$ (corresponding to a homogeneous aggregate of size $N=100), \Gamma_{10}=\gamma_{10} / 0.02$ and $\kappa=5 \mathrm{ps}^{-1}$. These values are consistent with measurements at low temperatures [13]-[14] and allows direct comparison with previous CPO works [15]. The transition dipole moment between $|0\rangle$ and $|1\rangle$ is $d_{10}^{1}=12.1$ $\mathrm{D}$ and the concentration of aggregates is $N_{0} \sim 10^{23} \mathrm{~m}^{-3}$. The dipole moment and the spontaneous emission of the transition between $|1\rangle$ and $|2\rangle$ are taken as $d_{21}=\sqrt{1.5} d_{10}$ and $\gamma_{21}=1.5 \gamma_{10}$. Here we have considered the average ratio of the oscillator strength of the relevant transitions as $f_{21} / f_{10} \sim d_{21}^{2} / d_{10}^{2} \sim 1.5$ [14]. Last, though to the best of our knowledge there is no experimental measurement of the rates $\gamma_{31}$ and $\gamma_{30}$, the employed values in our study are based on those found in the bibliography on exciton-exciton annihilation in J-aggregates [16]. For simplicity we take equal decay values $\gamma_{30}=\gamma_{31}=\gamma_{v} \gamma_{10}$. According to our simulations, relevant figures of merit for slow-light performance arise if $\left(\gamma_{30}+\gamma_{31}\right) \gtrsim \gamma_{10}$, so this will be the case in the following numerical study.

Figure 2 depicts the fractional delay and transmittance, calculated by integration of Eqs. (1) as a function of the modulation frequency $\delta$ and different values of $\gamma_{v}$. In this figure and hereafter, we only show results for the saturation intensity and $\gamma_{R} \lesssim \gamma_{R}^{*}$, where CPO-based slow light attains maximum delay [5]. If $\gamma_{R}<\gamma_{R}^{*}$ the fractional delay is reduced, while for $\gamma_{R}>\gamma_{R}^{*}$ the system shows bistability, which introduces a large distortion of the output signal. Notice that $\gamma_{R}^{*}$ depends on $\gamma_{v}$. Figure 2(a) shows that the particular value of $\gamma_{v}$ have no significant effect on the achieved maximum fractional delay ( $\mathrm{F} \sim 0.2)$. However, an increasing value of $\gamma_{v}$ shifts the optimum frequency to higher values up to $\sim 35 \mathrm{GHz}$ for $\gamma_{v}=3$. Figure 2(b) shows an increasing transmission with larger $\gamma_{v}$ as well. As it was already shown for the one-exciton approximation [5], there is gain in the weak sinusoidal modulation due to energy transfer between 
the background field and the sideband. This effect represents a remarkable advantage in contrast with the undesired residual absorption present in other CPO-based slow-light devices.

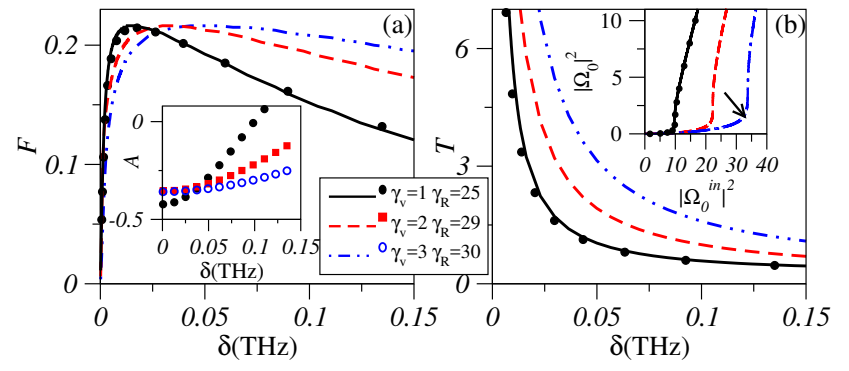

Fig. 2. (Color online) Fractional delay (a) and transmittance (b) of a sinusoidal input signal as a function of the modulation frequency $\delta$ for different values of the ratio $\gamma_{v}$ in absence of disorder. Inset (a) shows the absorption hole of the weak field. Inset (b) presents the output-input curve for the strong field $\Omega_{0}^{2}$, with the arrow pointing the saturation intensity for $\gamma_{v}=3$. Symbols result from analytical calculations based on Eqs. (4).

We now focus on the system response under illumination with a Gaussian-like pulse $\Omega=\Omega_{0}+\Omega_{m} \exp \left(\frac{-2 \sqrt{\log (2)} t}{\text { FWHM }}\right)^{2}$. Here FWHM refers to the full width half maximum of the temporal pulse. In this case, not only the attainable delay, but also distortion of the output pulses is relevant for applications. We define the distortion $D$ as the ratio between the output and input-pulse standard deviations. Figure 3 shows the fractional delay and distortion as a function of the input pulse temporal width. It can be seen that the pulse with maximum delay exhibits a large distortion. However, imposing a limit of $D=2$ (standard in slow-light experiments), values up to $F=0.4-0.5$ are obtained for 70 ps-long pulses, which gives rise to a $\sim 14$ GHz-bandwidth. As mentioned before, increasing values of $\gamma_{v}$ blue-shift the maximum delay and reduce the optimal pulse width. Lower distortions can be obtained by reducing the incident intensity below the optimal one. This also reduces the delay although it remains in a relevant order of magnitude for slow-light performance as shown in Fig. 3 with symbols. Inset of Fig. 3 presents how the signal transmittance is larger than 1 for most of the input-pulse widths analyzed, in agreement with Fig. 2. These results show that two-exciton dynamics drive to larger fractional delays for similar values of distortion in comparison to the two-level approximation.

In order to gain insight into the previous results, we turn into a more tractable model by assuming the following approximations in Eqs. (1). We neglect atomic coherence effects by eliminating $\sigma_{20}$ for $\dot{\sigma}_{10}$ and $\dot{\sigma}_{21}$. We also neglect the population $\rho_{22} \simeq 0$ since the exciton-exciton annihilation time is much faster than the rest of the decay times. Then, we adiabatically eliminate the coherences $\sigma_{10}$ and $\sigma_{21}$, as usual in CPO studies, since dephasing times are much shorter than the population decay times. Last, we also consider that the frequency of the incident field nearly matches the frequencies of the two relevant transitions. In the following we will show that the resulting system retains the fundamental features of the full four-level model of Eqs. (1).
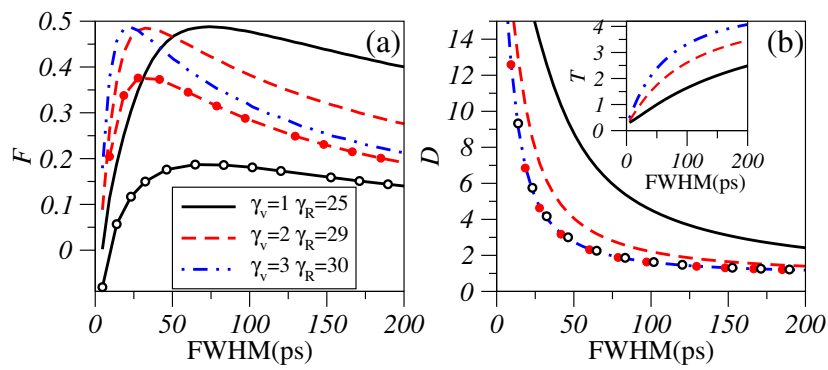

Fig. 3. (Color online) Fractional delay (a), distortion (b) and (inset) transmittance for pulsed input signals against the initial pulse temporal width (FWHM) in absence of disorder. Different values of $\gamma_{v}$ are considered. Lines with empty dots $\left(\gamma_{v}=1\right)$ and solid dots $\left(\gamma_{v}=2\right)$ show delays for incident intensities below the saturation point which reproduce the constrained distortion obtained for $\gamma_{v}=3$.

This simplified model reads,

$$
\begin{aligned}
\dot{\rho}_{11} & =-\gamma_{10} \rho_{11}+\gamma_{31} \rho_{33}-\frac{\mu_{21}^{2} \Gamma_{10}^{2} \Omega^{2}}{2 \Gamma_{21}+w} \rho_{11} \\
& -\frac{\mu_{10}^{2} \Gamma_{10} \Omega^{2}\left(2 \rho_{11}+\rho_{33}-1\right)}{2}, \\
\dot{\rho}_{33} & =-\left(\gamma_{30}+\gamma_{31}\right) \rho_{33}+\frac{\mu_{21}^{2} \Gamma_{10}^{2} \Omega^{2}}{2 \Gamma_{21}+w} \rho_{11} \\
\frac{\Omega^{i n}}{\Omega} & =1-\gamma_{R}\left(\mu_{10}^{2}\left(2 \rho_{11}+\rho_{33}-1\right)-\frac{2 \mu_{21}^{2} \Gamma_{10}}{2 \Gamma_{21}+w} \rho_{11}\right)
\end{aligned}
$$

Similarly to what was studied in Fig. 2, we consider a sinusoidally modulated incident field $\Omega=\Omega_{0}+\Omega_{m} \exp (-i \delta t)+$ c.c. that induces a periodic modulation of the populations at the beat frequency $\delta$, i.e. $\rho_{j j}=\rho_{j j}^{0}+\rho_{j j}^{m} \exp (-i \delta t)+$ c.c.. The coherent population oscillation modifies the absorption of the sidebands which leads to slow/fast light propagation. We insert this expansion in Eqs. (4) and equate terms oscillating at the same harmonic of $\delta$. The 0 -order term gives us the behavior of the strong field $\Omega_{0}$, see inset of Fig. 2 (b). The 1st-oder term in $\delta$ gives us the amplitude of the population oscillation and the sidebands fields. Then, we compute the transmission and delay time suffered by the sideband. Figure 2 (a) and (b) shows a good agreement between the full integration of Eqs. (1) and the analytical results given by this simplified model. The imaginary part of the susceptibility $\chi$ at the modulation frequency $\delta$ can also be easily obtained from Eqs. (4). Inset of Fig. 2 (a) depicts the characteristic hole in absorption present in CPO processes $A=\operatorname{Im}\left(\chi_{m}\right) / \operatorname{Im}\left(\chi_{0}\right)$. It can be seen how it broadens as $\gamma_{v}$ increases, which explains the blue-shift found in the maximum delay (see Figs. 2 and 3). To finish the study of the ordered system, we study the maximum fractional delay $F_{o p t}$ (versus $\delta$ ) by increasing the parameter $\gamma_{v}$ and the input Rabi frequency, see Fig. 4 (a). Figure 4 (b) shows $F_{o p t}$ as a function of the input intensity for $\gamma_{v}=3$. Two local maxima of $F_{o p t}$ can be seen for each value of $\gamma_{v}$. The first at lower input intensities relates to creation of one exciton in the aggregate and $\delta_{\text {opt }} \sim \gamma_{10}$ (not shown). Higher input intensities allow generation/annihilation of two excitons to play a role. The characteristic times of population dynamics are $\gamma_{10}$ and $\left(\gamma_{30}+\gamma_{31}\right)$. The latter could take higher values than $\gamma_{10}$, giving rise to a larger bandwidth with lower distortion, see Fig. 3. Moreover, by analyzing Eqs. (4) it can be demonstrated that such an optimal functionality could 
be achieved for $\gamma_{R} \lesssim 33$. This collective parameter can be obtained by modifying the temperature or increasing the aggregate concentration within reasonable experimental conditions.

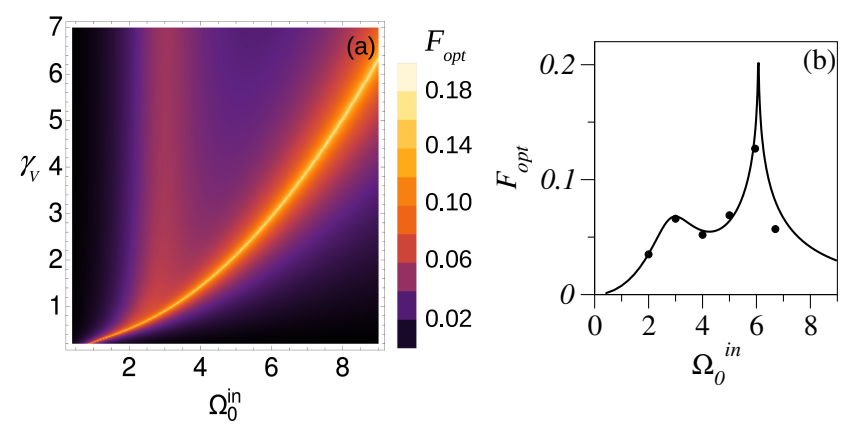

Fig. 4. (Color online) (a) Density plot shows the maximum fractional delay obtained from Eqs. (4) as a function of the ratio $\gamma_{v}$ and the input strong field $\Omega_{0}^{i n}$. (b) Right plot represents a comparison between the simplified model (solid line) and the full integration of Eqs. (1) (dots) for $\gamma_{v}=3$.

Last we analyze the effects of size dispersion on the slowlight performance for input pulsed signals. As mentioned before, the disordered aggregate is formed by coherent segments of different length N. In Ref. [12] it was demonstrated that such disorder effects can be modeled by a continuous distribution of transition energies $\omega_{10}^{N}$, while the dependency on $N$ of the rest of magnitudes can be neglected. Thus, such $N$-dependency is introduced into the detunings $\Delta_{10}^{N}$ and $\Delta_{21}^{N}$ in Eqs. (1). In our study we integrate Eqs. (1) for every coherent segment to calculate its contribution to the electric polarization in Eq. (2). Finally, to obtain the total molecular field we average such terms with the following Gaussian distribution:

$$
\sum_{N} p(N) \rightarrow \int_{-\infty}^{\infty} \frac{\exp \left(\frac{-\left(\Delta_{10}^{N}-\Delta_{10}\right)^{2}}{2 G^{2}}\right)}{\sqrt{2 \pi G^{2}}} d \Delta_{10}^{N} .
$$

Here, $G$ denotes the magnitude of the J-bandwidth resulting from the inhomogeneous broadening in units of $\Gamma_{10}$ and $\Delta_{10}$ refers to the detuning respect to the central frequency of the Gaussian. Similarly to the two-level approximation, the maximum attainable delays are reduced when $G$ is increased. However, we show that this nondesirable effect is much more constrained in the current four-level model, see Fig. 5 (a). For
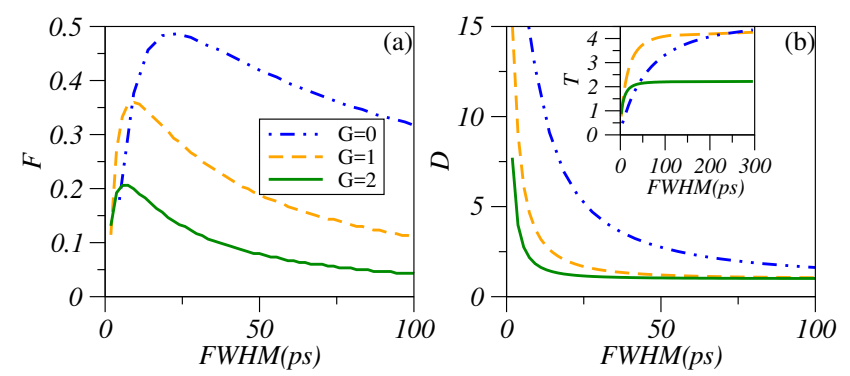

Fig. 5. (a) Fractional delay, (b) distortion and (inset) transmittance for pulsed input signals against the initial pulse temporal width (FWHM) for the ratio $\gamma_{v}=3$. Different magnitudes of disorder $G$ are considered.

example, a magnitude of disorder $G=2$ reduces the fractional delay up to 2.5 times with respect to the value obtained without size dispersion. However, this reduction is lower than that found in the two-level approximation where delays resulted 4 times lower [5]. Moreover, similarly to what was shown in that work, the detrimental effect of a larger inhomogeneous linewidth can be compensated by increasing the value of $\gamma_{R}$. More importantly, as a new feature of the current four-level model, the presence of disorder results in a remarkable blue-shift of the pulse bandwidth susceptible of slow-light performance up to values close to $100 \mathrm{GHz}$. In such a case the delay is still relevant for applications, $F \simeq 0.2$ for $G=2$, while keeping $D \leqq 2$.

Finally we concern about de aggregates photostability under the considered illumination conditions. For example, taking the optimum Rabi frequency inside the film $\Omega_{0} \sim 1.7 \Gamma_{10}$ for $\gamma_{R}=30$ and a pulse-width of 70 ps, we obtain an intensity of $1.3 \times 10^{14}$ photons $/ \mathrm{cm}^{2}$ per pulse in the sample. This value is clearly below the damage threshold in experiments [17] $\left(\sim 10^{16}\right.$ photons $/ \mathrm{cm}^{2}$ per pulse) so photobleaching is not expected to occur in the proposed optical device.

In summary we have analyzed CPO-based slow light in a Jaggregate nanofilm under pulse intensities high enough to excite two-exciton dynamics. It has been shown that fractional delays up to $\sim 0.5$ with constrained distortion can be obtained for pulse bandwidths of $\sim 14 \mathrm{GHz}$. Size dispersion significatively increases this available bandwidth up to values close to $100 \mathrm{GHz}$ maintaining a fractional delay close to 0.2 . These figures of merit support J-aggregates as a promising candidate for the design of slow-light devices at the nanoscale.

\section{Funding. MINECO (MAT2013-46308, FIS2013-41709-P).}

\section{REFERENCES}

1. J. Mørk, R. Kjær, M. van der Poel, and K. YvindOpt. Express 13, 8136 (2005).

2. K.-H. Kim, A. Husakou, and J. HerrmannOpt. Express 20, 25790 (2012).

3. S. Ek, P. Lunnemann, Y. Chen, E. Semenova, K. Yvind, and J. MorkNature communications 5 (2014).

4. T. Baba, H. C. Nguyen, N. Yazawa, Y. Terada, S. Hashimoto, and T. WatanabeScience and Technology of Advanced Materials 15, 024602 (2014).

5. E. Cabrera-Granado, E. Díaz, and O. G. CalderónPhys. Rev. Lett. 107, 013901 (2011).

6. J. KnoesterInternational Journal of Photoenergy 2006, 61364 (2006).

7. S. K. Saikin, A. Eisfeld, S. Valleau, and A. Aspuru-GuzikNanophotonics 2, 21 (2013).

8. H. Fidder, J. Knoester, and D. A. WiersmaThe Journal of Chemical Physics 98, 6564 (1993).

9. H. Glaeske, V. A. Malyshev, and K.-H. FellerPhys. Rev. A 65, 033821 (2002).

10. F. Herrera, B. Peropadre, L. A. Pachon, S. K. Saikin, and A. AspuruGuzikThe Journal of Physical Chemistry Letters 5, 3708 (2014). PMID: 26278740.

11. G. Zengin, M. Wersäll, S. Nilsson, T. J. Antosiewicz, M. Käll, and T. ShegaiPhys. Rev. Lett. 114, 157401 (2015).

12. E. C. Jarque and V. A. MalyshevThe Journal of Chemical Physics 115 (2001).

13. H. Fidder, J. Knoester, and D. A. WiersmaChemical Physics Letters 171, 529 (1990).

14. K. Minoshima, M. Taiji, K. Misawa, and T. KobayashiChemical Physics Letters 218, 67 (1994).

15. R. W. Boyd, M. G. Raymer, P. Narum, and D. J. HarterPhys. Rev. A 24, 411 (1981).

16. H. Stiel, S. Daehne, and K. TeuchnerJournal of Luminescence 39, 351 (1988).

17. R. V. Markov, Z. M. Ivanova, A. I. Plekhanov, N. A. Orlova, and V. V. ShelkovnikovQuantum Electronics 31, 1063 (2001). 


\section{FULL REFERENCES}

1. J. Mørk, R. Kjær, M. van der Poel, and K. Yvind, "Slow light in a semiconductor waveguide at gigahertz frequencies," Opt. Express 13, 8136-8145 (2005).

2. K.-H. Kim, A. Husakou, and J. Herrmann, "Slow light in dielectric composite materials of metal nanoparticles," Opt. Express 20, 2579025797 (2012).

3. S. Ek, P. Lunnemann, Y. Chen, E. Semenova, K. Yvind, and J. Mork, "Slow-light-enhanced gain in active photonic crystal waveguides," $\mathrm{Na}$ ture communications 5 (2014).

4. T. Baba, H. C. Nguyen, N. Yazawa, Y. Terada, S. Hashimoto, and T. Watanabe, "Slow-light mach-zehnder modulators based on si photonic crystals," Science and Technology of Advanced Materials 15, 024602 (2014).

5. E. Cabrera-Granado, E. Díaz, and O. G. Calderón, "Slow light in molecular-aggregate nanofilms," Phys. Rev. Lett. 107, 013901 (2011).

6. J. Knoester, "Modeling the optical properties of excitons in linear and tubular j-aggregates," International Journal of Photoenergy 2006, 61364 (2006).

7. S. K. Saikin, A. Eisfeld, S. Valleau, and A. Aspuru-Guzik, "Photonics meets excitonics: natural and artificial molecular aggregates," Nanophotonics 2, 21-38 (2013).

8. H. Fidder, J. Knoester, and D. A. Wiersma, "Observation of the oneexciton to two-exciton transition in a j aggregate," The Journal of Chemical Physics 98, 6564-6566 (1993).

9. H. Glaeske, V. A. Malyshev, and K.-H. Feller, "Mirrorless optical bistability of an ultrathin glassy film built up of oriented j-aggregates: Effects of two-exciton states and exciton-exciton annihilation," The Journal of Chemical Physics 114, 1966-1969 (2001).

10. F. Herrera, B. Peropadre, L. A. Pachon, S. K. Saikin, and A. AspuruGuzik, "Quantum nonlinear optics with polar j-aggregates in microcavities," The Journal of Physical Chemistry Letters 5, 3708-3715 (2014). PMID: 26278740 .

11. G. Zengin, M. Wersäll, S. Nilsson, T. J. Antosiewicz, M. Käll, and T. Shegai, "Realizing strong light-matter interactions between singlenanoparticle plasmons and molecular excitons at ambient conditions," Phys. Rev. Lett. 114, 157401 (2015).

12. A. Eisfeld and J. Briggs, "The shape of the j-band of pseudoisocyanine," Chemical Physics Letters 446, 354 - 358 (2007).

13. H. Fidder, J. Knoester, and D. A. Wiersma, "Superradiant emission and optical dephasing in j-aggregates," Chemical Physics Letters 171, 529 -536 (1990).

14. K. Minoshima, M. Taiji, K. Misawa, and T. Kobayashi, "Femtosecond nonlinear optical dynamics of excitons in j-aggregates," Chemical Physics Letters 218, 67 - 72 (1994).

15. R. W. Boyd, M. G. Raymer, P. Narum, and D. J. Harter, "Four-wave parametric interactions in a strongly driven two-level system," Phys. Rev. A 24, 411-423 (1981).

16. R. V. Markov, Z. M. Ivanova, A. I. Plekhanov, N. A. Orlova, and V. V. Shelkovnikov, "Resonance absorber based on thin films of pseudoisocyanine j-aggregates," Quantum Electronics 31, 1063 (2001). 